\title{
SUM-FREE SETS OF INTEGERS
}

\author{
H. L. ABBOTT ${ }^{1}$ AND E. T. H. WANG ${ }^{2}$
}

\begin{abstract}
A set $S$ of integers is said to be sum-free if $a, b \in S$ implies $a+b \notin S$. In this paper, we investigate two new problems on sum-free sets: (1) Let $f(k)$ denote the largest positive integer for which there exists a partition of $\{1,2, \ldots, f(k)\}$ into $k$ sum-free sets, and let $h(k)$ denote the largest positive integer for which there exists a partition of $\{1,2, \ldots, h(k)\}$ into $k$ sets which are sum-free mod $h(k)+1$. We obtain evidence to support the conjecture that $f(k)=h(k)$ for all $k$. (2) Let $g(n, k)$ denote the cardinality of a largest subset of $\{1,2, \ldots, n\}$ that can be partitioned into $k$ sum-free sets. We obtain upper and lower bounds for $g(n, k)$. We also show that $g(n, 1)=[(n+1) / 2]$ and indicate how one may show that for all $n<54, g(n, 2)=n-[n / 5]$.
\end{abstract}

A set $S$ of integers is said to be sum-free if $a, b \in S$ implies $a+b \notin S$. The case $a=b$ is not excluded, so that $a \in S$ implies $2 a \notin S$. A well-known theorem of I. Schur [14] states that if the set $\{1,2, \ldots,[k ! e]\}$ is partitioned arbitrarily into $k$ sets, at least one of the sets fails to be sum-free. Thus we may define $f(k)$ to be the largest positive integer for which there exists some way of partitioning $\{1,2, \ldots, f(k)\}$ into $k$ sum-free sets.

The determination of the numbers $f(k)$ is a notoriously difficult problem. It is easy to verify that $f(1)=1, f(2)=4$ and with a little effort one can show that $f(3)=13$. L. D. Baumert [4], with the aid of a computer, showed that $f(4)=44$. The value of $f(5)$ seems to be out of reach at the present time. Recently H. Fredericksen [9] proved that $f(5) \geqslant 138$ and this seems to be the record.

Schur proved that $f(k+1) \geqslant 3 f(k)+1$ and this, together with his theorem mentioned in the first paragraph, shows that

$$
\left(3^{k}-1\right) / 2 \leqslant f(k) \leqslant[k ! e]-1 .
$$

Abbott and Hanson [3], improving on an earlier inequality of Abbott and Moser [2], proved that for all positive integers $k$ and $l$,

$$
f(k+l) \geqslant 2 f(k) f(l)+f(k)+f(l) .
$$

From (2), or the earlier inequality of Abbott and Moser, it follows, via a well-known argument, that $\alpha=\lim _{k \rightarrow \infty} f(k)^{1 / k}$ exists, although $\alpha$ may be

Received by the editors December 20, 1976 and, in revised form, April 29, 1977.

AMS (MOS) subject classifications (1970). Primary $10 \mathrm{~L} 10$.

Key words and phrases. Sum-free set, $k$-wise sum-free set.

'Supported by the National Research Council of Canada under grant NRC A-2971.

${ }^{2}$ Supported by the National Research Council of Canada under grant NRC A-9121 and by a Summer Research Fellowship from Wilf rid Laurier University. 
infinite. Schur's lower bound shows that $\alpha \geqslant 3$. The result of Baumert, with (2), gives $\alpha \geqslant(89)^{1 / 4}=3.0714 \ldots$, while Fredericksen's lower bound for $f(5)$ gives $\alpha \geqslant(277)^{1 / 5}=3.0796 \ldots$ The upper bound given in (1) has been improved slightly by $\mathrm{R}$. W. Irving [11] to $f(k) \leqslant\left[k !\left(e-\frac{1}{24}\right)\right]$ by appealing to a result of E. G. Whitehead [16] on Ramsey numbers. An account of most of these results may be found in the article by A. P. Street [15]. See also the interesting paper of L. Mirsky [13].

In this paper we investigate two new problems on sum-free sets.

Problem I. Denote by $h(k)$ the largest integer $m$ for which there exists some way of partitioning $\{1,2, \ldots, m\}$ into $k$ sets which are sum-free modulo $m+1$; that is, which contain no solution of $x+y \equiv z(\bmod m+1)$. The partitions which establish the values $f(1)=1, f(2)=4, f(3)=13, f(4)=44$ are sum-free modulo $2,5,14,45$, respectively, and thus $h(k)=f(k)$ for $k \leqslant 4$. This suggests the conjecture $h(k)=f(k)$ for all $k$. We have not been able to prove this conjecture but we obtain some evidence to support it by showing that $h$ satisfies the same recurrence inequality (2) as $f$.

Problem II. We say that a set of integers is $k$-wise sum-free (or $k$-SF) if it can be partitioned into $k$ sum-free sets. Let $n$ be a positive integer and let $N_{n}=\{1,2, \ldots, n\}$. Denote by $g(n, k)$ the size of a largest $k$-SF subset of $N_{n}$. It is not hard to evaluate $g(n, 1)$. In this paper we indicate how one may evaluate $g(n, 2)$ for $n \leqslant 54$ and obtain some general upper and lower bounds for $g(n, k)$.

THEOREM 1. For all positive integers $k$ and $l$,

$$
h(k+l) \geqslant 2 h(k) h(l)+h(k)+h(l) .
$$

Proof. The argument parallels closely the proof of (2) as given in [1]. Partition $\{1,2, \ldots, h(k)\}$ into sets $A_{1}, A_{2}, \ldots, A_{k}$ which are SF $\bmod (h(k)$ $+1)$ and partition $\{1,2, \ldots, h(l)\}$ into sets $B_{1}, B_{2}, \ldots, B_{l}$ which are SF $(\bmod h(l)+1)$. For $1 \leqslant i \leqslant k$ let

$$
C_{i}=\left\{s(2 h(k)+1)+t: s=0,1, \ldots, h(l), t \in A_{i}\right\},
$$

and for $k<i \leqslant k+l$ let

$$
C_{i}=\left\{s(2 h(k)+1)-t: s \in B_{i-k}, t=0,1, \ldots, h(k)\right\} .
$$

Then it is easy to check that $C_{i} \cap C_{j}=\varnothing$ if $i \neq j$ and that $\cup_{i=1}^{k+l} C_{i}=\{1$, $2, \ldots, m-1\}$ where $m=2 h(k) h(l)+h(k)+h(l)+1$. We claim that $C_{i}$ is $\mathrm{SF}(\bmod m)$.

Suppose that for some $i, 1 \leqslant i \leqslant k$, there are numbers $a, b, c \in C_{i}$ such that

$$
a+b \equiv c \quad(\bmod m)
$$

We have

$$
\begin{gathered}
a=s_{a}(2 h(k)+1)+t_{a}, \quad b=s_{b}(2 h(k)+1)+t_{b}, \\
c=s_{c}(2 h(k)+1)+t_{c}
\end{gathered}
$$


where $0 \leqslant s_{a}, s_{b}, s_{c} \leqslant h(l)$ and $t_{a}, t_{b}, t_{c} \in A_{i}$. It is easy to check that $-m+3 \leqslant a+b-c \leqslant 2 m-3$ and, hence, in order for (3) to hold, we must have

$$
a+b-c=r m \text { where } r=0 \text { or } 1 .
$$

It follows from (4) and (5) that

(6) $\left(s_{a}+s_{b}-s_{c}-r h(l)\right)(2 h(k)+1)=r(h(k)+1)-t_{a}-t_{b}+t_{c}$.

The right side of (6) lies between $-2 h(k)+1$ and $2 h(k)-1$ and must therefore be zero. It follows that $t_{a}+t_{b}=t_{c}+r(h(k)+1)$, and this contradicts the fact that $A_{i}$ is $\mathrm{SF}(\bmod (h(k)+1))$.

A similar contradiction arises in case $k<i \leqslant k+l$, the only difference being that one gets, instead of (6),

$$
\left(s_{a}+s_{b}-s_{c}-r(h(l)+1)\right)(2 h(k)+1)=t_{a}+t_{b}-t_{c}-r h(k) .
$$

The right side of (7) lies between $-2 h(k)$ and $2 h(k)$ and must therefore be zero. Thus $s_{a}+s_{b}=s_{c}=r(h(l)+1)$, contradicting the fact that $B_{i-k}$ is $\mathrm{SF}(\bmod h(l)+1)$. This completes the proof.

Corollary 1. $\beta=\lim _{k \rightarrow \infty} h(k)^{1 / k}$ exists.

REMARK 1. The corollary follows easily from Theorem 1 via a well-known argument which we do not give here (see, for example, [2]). It would be of interest to know whether $\alpha=\beta$. We point out that the partition given by Fredericksen to show that $f(5) \geqslant 138$ is $\operatorname{SF}(\bmod 139)$. Thus $\beta \geqslant(277)^{1 / 5}$.

We now discuss Problem II. It is clear that $g(n, k)$ is a nondecreasing function of $k$, and that if $n \leqslant f(k)$, then $g(n, k)=n$. It is not hard to evaluate $g(n, 1)$. We state this as a theorem.

THEOREM 2. $g(n, 1)=[(n+1) / 2]$.

Proof. Let $A=\left\{a_{1}, a_{2}, \ldots, a_{t}\right\} \subseteq N_{n}$ be a SF set, $1 \leqslant a_{1}<a_{2}<\cdots<$ $a_{t}$. Then the $2 t-1$ numbers $a_{1}, a_{2}, \ldots, a_{t}, a_{t}-a_{1}, a_{t}-a_{2}, \ldots, a_{t}-a_{t-1}$ are distinct and belong to $N_{n}$. Thus $2 t-1 \leqslant n$ so that $g(n, 1) \leqslant[(n+1) / 2]$. Since the set $O_{n}$ of odd integers in $N_{n}$ is SF and has $[(n+1) / 2]$ elements, we have $g(n, 1)=[(n+1) / 2]$.

REMARK 2. $O_{n}$ is not the unique largest SF subset of $N_{n}$, since if $n=2 t$, the set $\{t+1, t+2, \ldots, 2 t\}$ is SF and if $n=2 t+1$, the set $\{t+1, t+$ $2, \ldots, 2 t+1\}$ is SF.

REMARK 3. It is interesting to note that if we consider any set of $n$ integers, not necessarily $N_{n}$, then it may not be possible to select a SF subset with $[(n+1) / 2]$ elements, e.g., consider the set $\{2,3,4,5,6,8,10\}$. (This example is due to D. Klarner and is quoted in [8].) In fact, if one denotes by $\phi(n)$ the largest integer such that every set of $n$ real numbers, different from zero, always contains a SF subset with $\phi(n)$ elements, then it is is known [8] that $n / 3 \leqslant \phi(n) \leqslant 3 n / 7$. Other bounds for $\phi(n)$ have been obtained by Erdös, Choi, Komlós, Sulyok and Szemerédi [5]-[8], [12]. Erdös also mentioned that $\phi(n)=[(n+2) / 2]$ is likely if we exclude sums of equal summands. 
THEOREM 3. $g(n, k) \geqslant n-[n /(h(k)+1)]$.

Proof. Partition $\{1,2, \ldots, h(k)\}$ into sets $A_{1}, A_{2}, \ldots, A_{k}$ which are SF $(\bmod h(k)+1)$ and let

$$
B_{i}=\left\{b: 1 \leqslant b \leqslant n, b \equiv a(\bmod h(k)+1) \text { for some } a \in A_{i}\right\} .
$$

Then it is clear that $B_{i}$ is SF and the theorem follows.

COROllaRY 2. $g(n, 2) \geqslant n-[n / 5], g(n, 3) \geqslant n-[n / 14], g(n, 4) \geqslant n-$ [n/45].

We conjecture that equality holds in Theorem 3, but we have not been able to prove this, even in the case $k=2$. We now prove a lemma which enables us to show that $g(n, 2)=n-[n / 5]$ for $n \leqslant 54$.

LEMMA 1. For positive integers $a$ and $b$ such that $b+4 a \leqslant n$, let $P_{a, b}=\{b$, $b+a, b+2 a, b+3 a, b+4 a\}$ and $Q_{a, b}=\{a, 2 a\} \cup P_{a, b}$. For positive integers $a$ and $b$ such that $a<b$ and $2 b+a \leqslant n$, let $R_{a, b}=\{a, b, 2 b, b \pm a$, $2 b \pm a\}$. Let $N_{n}=A \cup B \cup C$ be a partition where $A$ and $B$ are $S F$. Then

(i) $C \cap Q_{a, b} \neq \varnothing$;

(ii) if $a \notin C$ and $2 a \notin C$ then $C \cap P_{a, b} \neq \varnothing$;

(iii) $C \cap R_{a, b} \neq \varnothing$.

Proof. (i) Suppose $C \cap Q_{a, b}=\varnothing$. Then $a \notin C$ and we may suppose without loss of generality that $a \in A$. Then $2 a \in B$. If $b \in B$ then $b+2 a \in$ $A$ and $b+3 a \in B$. Thus $b+a \notin A \cup B$, so that $b+a \in C$, a contradiction. Thus $b \in A$. But then $b+a \in B, b+3 a \in A$ and $b+4 a \in B$, so that $b+2 a \notin A \cup B$. Thus $b+2 a \in C$, a contradiction. This proves (i).

(ii) Follows immediately from (i).

(iii) Suppose $C \cap R_{a, b}=\varnothing$. Let $a \in A$, say. If $b \in A$, we have $b \pm a \in B$ so that $2 b \notin A \cup B$, a contradiction. Thus $b \in B$. Then $2 b \in A, 2 b \pm a \in$ $B$ and, hence, $b \pm a \in A$, which again gives a contradiction. This completes the proof of the lemma.

The lemma, simple though it is, enables us to narrow considerably the number of possibilities for $C$. To illustrate, we evaluate $g(20,2)$.

THEOREM 4. $g(20,2)=16$.

Proof. Suppose not. Then there exists a partition $N_{20}=A \cup B \cup C$ where $A$ and $B$ are SF and $|C|=3$. By Lemma 1(i), $C \cap Q_{3,3} \neq \varnothing$ and $C \cap Q_{4,4} \neq \varnothing$ and, since $Q_{3,3} \cap Q_{4,4}=\{12\}, C$ cannot contain both 1 and 2 . For otherwise, $C=\{1,2,12\}$ and hence $C \cap R_{3,7}=\varnothing$, contradicting (iii). Hence there are three cases to be considered. We arrive at a contradiction in each case.

Case 1. $1 \notin C, 2 \notin C$. Then $C$ meets each of $P_{1,6}, P_{1,11}$ and $P_{1,16}$. Since these three sets are pairwise disjoint, $C$ must be contained in their union. Hence $C \cap Q_{1,1}=\varnothing$, contradicting (i).

Case 2. $1 \in C, 2 \notin C$. If $4 \notin C$, then $C$ meets each of the sets $P_{2,2}, P_{2,3}$ and 
$P_{2,12}$. Since these three sets are pairwise disjoint and none of them contains 1 , we get $|C| \geqslant 4$, a contradiction. Hence $4 \in C$. If $3 \notin C$ and $6 \notin C$ then $C$ meets $P_{3,2}$ and $P_{3,3}$. Since these two sets are disjoint and neither contains 1 or 4, we get $|C| \geqslant 4$, a contradiction. Thus $C=\{1,3,4\}$ or $\{1,4,6\}$. In either case $C \cap R_{2,7}=\varnothing$, which contradicts (iii).

Case 3. $1 \notin C, 2 \in C$. If $3 \notin C$ and $6 \notin C$ then $C$ meets each of the sets $P_{3,1}, P_{3,3}$ and $P_{3,5}$. Since these sets are pairwise disjoint and none of them contains 2, we get $|C| \geqslant 4$, a contradiction. Thus $3 \in C$ or $6 \in C$. If $4 \notin C$ and $8 \notin C$, then since $C$ meets each of the sets $P_{4,1}, P_{4,3}$ and $P_{4,4}$, we again get a contradiction. Thus $4 \in C$ or $8 \in C$. Thus $C$ is one of the sets $\{2,3,4\}$, $\{2,3,8\},\{2,4,6\},\{2,6,8\}$. But $\{2,3,4\} \cap R_{1,6}=\{2,3,8\} \cap R_{1,5}=\{2,4$, 6\} $\cap R_{1,8}=\{2,6,8\} \cap R_{3,4}=\varnothing$, contradicting Lemma 1(iii). This completes the proof.

REMARK 4. Using the same method we have verified that $g(n, 2)=n-$ [ $n / 5]$ for $n \leqslant 54$. For the larger values of $n$, however, the number of possibilities for $C$ becomes greater and the case-by-case argument becomes very tedious. It is possible that by using Baumert's algorithm [4], one may be able to evaluate $g(n, 2)$ for a few more values of $n$, but since it is unlikely that any insight into what happens for general $n$ will result, we have not looked into this.

We now obtain a general upper bound for $g(n, k)$. We write $m=f(k)+1$, and let

$$
\alpha_{k}=\liminf _{k \rightarrow \infty} \frac{g(n, k)}{n} \leqslant \limsup _{n \rightarrow \infty} \frac{g(n, k)}{n}=\beta_{k} .
$$

It is not hard to show that $\beta_{k} \leqslant 1-1 / \mathrm{m}^{2}$. We now indicate how this may be improved.

\section{THEOREM 5.}

$$
\beta_{k} \leqslant 1-\frac{1}{m} \prod_{p<m}\left(1-\frac{1}{p}\right)<1-\frac{c}{m \log m},
$$

where the product is taken over all primes $p \leqslant m$, and where $c$ is a positive constant.

Proof. Let $N_{n}=A_{1} \cup A_{2} \cup \cdots \cup A_{k} \cup C$ be a partition in which each $A_{i}$ is SF. For each $a$ such that $m a \leqslant n$ let $Q_{a}=\{a, 2 a, \ldots, m a\}$. It is clear that $C$ has nonempty intersection with each $Q_{a}$, since otherwise we would get a partition of $\{1,2, \ldots, m\}$ into $k$ sum-free sets. We now show that there is a large collection of $Q$ 's which are pairwise disjoint. If $a, b \leqslant n / m$, where $a \neq b$ and all prime factors of $a$ and $b$ exceed $m$, then $Q_{a} \cap Q_{b}=\varnothing$. Thus

$$
|C| \geqslant \mid\{a: a \leqslant n / m \text {, all prime factors of } a \text { exceed } m\} \mid \text {. }
$$

Let $P=\Pi_{p \leqslant m} p$ and let $\mu$ denote the usual Möbius function (see [10, p. 234]). Then, by the inclusion-exclusion principle (see [10, p. 234, Theorem 261]), and keeping in mind that all prime factors of 1 exceed $m$, we get 


$$
\begin{aligned}
|C| & \geqslant \sum_{d \mid P} \mu(d)\left[\frac{n}{m d}\right] \geqslant \frac{n}{m} \sum_{d \mid P} \frac{\mu(d)}{d}-\sum_{d \mid P} 1 \\
& =\frac{n}{m} \prod_{p<m}\left(1-\frac{1}{p}\right)-2^{\pi(m)},
\end{aligned}
$$

where $\pi(m)$ is the number of primes not exceeding $m$. It follows that

$$
\beta_{k} \leqslant 1-\frac{1}{m} \prod_{p<m}\left(1-\frac{1}{p}\right)<1-\frac{c}{m \log m},
$$

where the last inequality follows from the theorem of Mertens (see [10, p. 351]).

REMARK 5. It would be of interest to know whether Theorem 5 can be sharpened to $\beta_{k} \leqslant 1-c / m$, for some positive constant $c$. With regard to the problem of getting a lower bound for $\alpha_{k}$, we point out that we have not been able to do better than Theorem 3. If our conjecture that $h(k)=f(k)$ is true then, of course, $\alpha_{k} \geqslant 1-1 / m$. It would be of interest to prove an inequality of the form $\alpha_{k} \geqslant 1-c / m$ without appealing to any unproved conjecture, but we have not been able to do this.

\section{REFERENCES}

1. H. L. Abbott and D. Hanson, Lower bounds of certain types of van der Waerden numbers, J. Combinatorial Theory 12 (1972), 143-146.

2. H. L. Abbott and L. Moser, Sum-free sets of integers, Acta Arith. 11 (1966), 393-396.

3. H. L. Abbott and D. Hanson, On a problem of Schur and its generalizations, Acta Arith. 20 (1972), 175-187.

4. L. D. Baumert, Sum-free sets, J. P. L. Research Summary No. 36-10, 1 (1961), 16-18.

5. S. L. G. Choi, The largest sum-free subsequence from a sequence of $n$ numbers, Proc. Amer. Math. Soc. 39 (1973), 42-44.

6. On sequences not containing a large sum-free subsequence, Proc. Amer. Math. Soc. 41 (1973), 415-418.

7. S. L. G. Choi, J. Komlós and E. Szemerédi, On sum-free subsequences, Trans. Amer. Math. Soc. 212 (1975), 307-313.

8. P. Erdös, Extremal problems in number theory, Proc. Sympos. Pure Math., vol. 8, Amer. Math. Soc., Providence, R. I., 1965, pp. 181-189.

9. H. Fredericksen, Five sum-free sets, Proc. Sixth Annual Southeastern Conf. on Graph Theory, Combinatorics and Computing, 1975, pp. 309-314.

10. G. H. Hardy and E. M. Wright, An introduction to the theory of numbers, 4th ed., Oxford Univ. Press, London and New York, 1960.

11. R. W. Irving, An extension of Schur's theorem on sum-free partitions, Acta Arith. 25 (1973), $55-63$.

12. J. Komlós, M. Sulyok and E. Szemerédi, Linear problems in combinatorial number theory, Acta Math. Acad. Sci. Hungar.26 (1975), 113-121.

13. L. Mirsky, The combinatorics of arbitrary partitions, Bull. Inst. Math. Appl. 11 (1975), 6-9.

14. I. Schur, Über die Kongruenz $x^{m}+y^{m} \equiv z^{m}(\bmod p)$, Jber. Deutsch. Math.-Verein. 25 (1916), 114-117.

15. W. D. Wallis, A. P. Street and J. S. Wallis, Combinatorics: Room squares, sum-free sets, Hadamard matrices, Lecture Notes in Math., vol. 292, Springer-Verlag, Berlin and New York, 1972.

16. E. G. Whitehead,Jr., The Ramsey number $N(3,3,3,3, ; 2)$, Discrete Math. 4 (1973), 389-396.

Department of Mathematics, University of Alberta, Edmonton, Alberta, Canada

Department of Mathematics, Wilfrid laurier University, Waterloo, Ontario, Canada 